$12,14,19$

\title{
Теплопроводность цепочки ротаторов с двухбарьерным потенциалом взаимодействия
}

\author{
(C) А.П. Клинов, М.А. Мазо, В.В. Смирнов \\ Федеральный исследовательский центр химической фризики им. Н.Н. Семёнова РАН, \\ Москва, Россия \\ E-mail: artemklinov@gmail.com
}

Поступила в Редакцию 29 января 2021 г.

В окончательной редакции 20 марта 2021 г.

Принята к публикации 21 марта 2021 г.

\begin{abstract}
Представлены результаты численного моделирования теплопроводности одномерной цепи ротаторов с двухбарьерным потенциалом взаимодействия ближайших соседей. Показано, что величина „внутреннего“ барьера, разделяющего топологически неэквивалентные вырожденные состояния, существенным образом влияет на температурную зависимость теплопроводности цепи. При малых высотах этого барьера в области низких температур основной вклад в рост теплопроводности вносят нелинейные нормальные моды. С увеличением температуры рост теплопроводности ограничивается появлением локальных надбарьерных переходов, препятствующих переносу энергии вдоль цепи. При увеличении высоты „внутреннего“ барьера вклад нелинейных нормальных мод в процесс переноса энергии снижается и система демонстрирует температурное поведение, характерное для систем обычных ротаторов.
\end{abstract}

Ключевые слова: моделирование, одномерные системы, модель ротаторов, теплопроводность, ДНК-подобные полимеры.

DOI: 10.21883/FTT.2021.07.51051.016

\section{1. Введение}

Проблема переноса тепла в твердых диэлектриках в течение долгого времени была и, по-видимому, будет оставаться вызовом для специалистов, занимающихся физикой твердого тела. Даже в случае бездефектных монокристаллических образцов расчеты коэффициента теплопроводности не являются тривиальной задачей [1].

Первые численные эксперименты Э. Ферми, выполненные совместно с Д. Паста, С. Уламом и М. Тсингоу более полувека назад [2], положили начало новой междисциплинарной нелинейной науке [3]. Эти эксперименты привели к открытию новых типов волнового движения в одномерных системах - локализованным нормальным модам, играющим большую роль в современной картине физических явлений. В последние десятилетия эти скорее теоретические конструкции в виде нелинейных одномерных цепочек получили неожиданное практическое приложение после открытия однои двумерных наноструктур - углеродных нанотрубок и графена $[4,5]$.

Однако надо отметить, что задолго до этих революционных открытий нелинейные одномерные решетки использовались как модели для описания сильно анизотропных систем - полимерных кристаллов гибкоцепных полимеров, а также в приложении к исследованию ферромагнетиков [6]. Исследования в этой области привели к открытию ряда фундаментальных явлений [7], тем не менее проблема теплопроводности даже в одномерных модельных системах остается открытой. Этой проблеме посвящено множество статей и обзоров [8-10], в которых были исследованы различные типы взаимодействия частиц и/или различные граничные условия. Еще в первых численных экспериментах было показано, что коэффициент теплопроводности одномерных цепочек с ангармоническим потенциалом взаимодействия зависит от температуры и длины цепи и, как правило, расходится в пределе бесконечно длинных цепей. В центре внимания исследований находился вопрос о том, какой потенциал взаимодействия частиц приводит к конечной теплопроводности цепочки. Было показано, что цепочки с локальным потенциалом взаимодействия (потенциалом взаимодействия с подложкой) в большинстве случаев обладают конечной теплопроводностью [9]. В случае систем без локального потенциала ограниченность или асимметрия потенциалов взаимодействия не играют принципиальную роль в сходимости коэффициента теплопроводности с ростом числа частиц - важно, чтобы потенциал допускал определенные локальные возбуждения цепи, вызывающие рассеяние проходящих фононов.

Примерами такого рода возбуждений являются ротобризеры в цепочке ротаторов $[11,12]$ или локальные растяжения связей в случае цепочки частиц с леннардджонсовским потенциалом взаимодействия [13]. Некоторые исследователи также дополняют указанный список систем с конечной теплопроводностью цепочками $\beta$-ФПУ с двухъямным потенциалом взаимодействия [14]. Несмотря на то, что расчеты с помощью неравновесной молекулярной динамики указывают скорее на медленный рост коэффициента теплопроводности в области температур вблизи барьера [14-16], переходы между двумя ямами, по-видимому, вызывают сильное рассеяние 


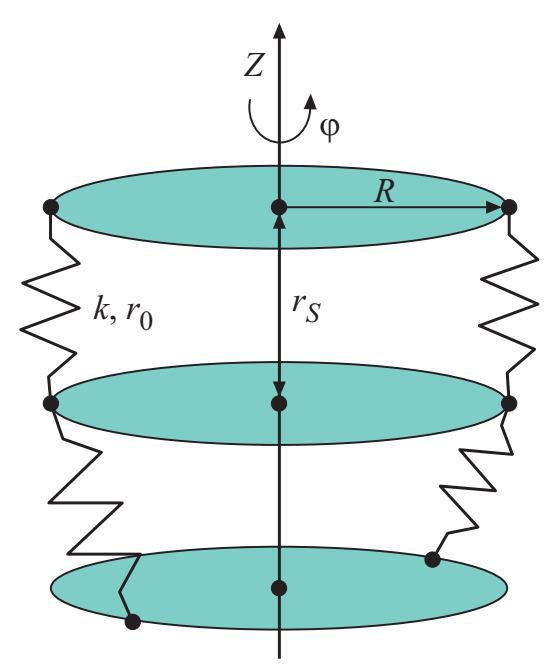

Рис. 1. Модель взаимодействующих дисков.

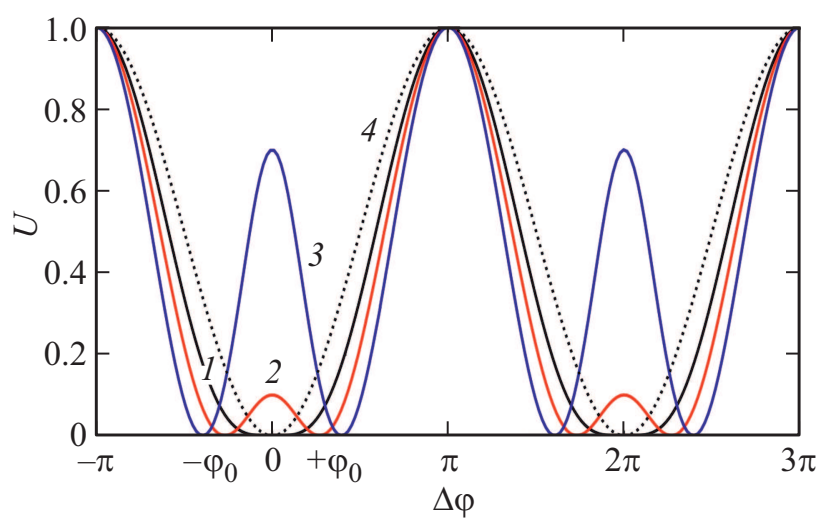

Рис. 2. Периодический двухбарьерный потенциал взаимодействия соседних мономеров при различных высотах внутреннего барьера, разделяющего вырожденные состояния $\pm \varphi_{0}$. Кривая 1 соответствует высоте внутреннего барьера $p=0$, $2-0.1,3-0.7$. Кривая 4 показывает потенциал, соответствующий физическому маятнику $U(x)=(1-\cos x) / 2$. Топологически эквивалентными состояниями являются все состояния $+\varphi_{0} \pm 2 \pi n$ или $-\varphi_{0} \pm 2 \pi n(n=1,2, \ldots)$.

фононов и замедляют рост коэффициента теплопроводности при увеличении длины цепи. Механизм снижения теплопроводности в системах с двухъямным потенциалом недостаточно изучен и представляет самостоятельную научную проблему.

Данная работа посвящена динамике одномерных цепей с периодическим двухбарьерным потенциалом. Как было показано в работе [12], именно цепочки ротаторов демонстрируют ограниченную теплопроводность в пределе бесконечно длинных цепей. Этим они отличаются от систем с неограниченными потенциалами и сохранением импульса (например, решетки ФПУ), в которых коэффициент теплопроводности обычно растет с увеличением длины цепи. С точки зрения приложений интересной является также возможность управления характеристиками такой системы путем изменения граничных условий [17].

Выбор двухбарьерного потенциала в данной работе обусловлен двумя факторами. Во-первых, в настоящее время активно обсуждаются модели искусственных двухспиральных молекул (ксенонуклеиновые кислоты [18]), торсионная подвижность которых может быть в первом приближении представлена системой соосных дисков [17]. Взаимодействие между соседними дисками обеспечивается двумя пружинами (см. рис. 1). Если расстояние между соседними дисками меньше, чем длина пружин, то минимум потенциальной энергии соответствует относительному повороту дисков на некоторый угол $\Delta \varphi=\varphi_{0}$ (рис. 2). Высота барьера, разделяющего положения равновесия, зависит от отношения длины пружины к расстоянию между дисками. Таким образом, изменение данного параметра позволяет управлять динамическими характеристиками системы.

Важнейшим свойством гибкоцепных полимеров является их конформационная подвижность и наличие транси гош-конформаций. Однако большинство работ, описывающих нелинейную динамику полимерных цепей, было выполнено, фактически, в квазилинейном малоамплитудном приближении, в котором не учитываются различные устойчивые конформации цепи $[19,20]$. Очевидно, что при больших температурах такое приближение недостаточно, поскольку в системе с двойным вырождением основного состояния при конечных температурах должно наблюдаться разупорядочение цепи, что приводит к существенному изменению спектра. Таким образом, хотя в реальных полимерах транс- и гош-конформации энергетически не вырождены, наличие второго минимума должно существенным образом сказываться на динамике системы и, как следствие, на ее тепловых характеристиках.

\section{2. Модель}

Рассмотрим одномерную систему соосных дисков с радиусом $R$, расположенных с шагом $r_{s}$ (см. рис. 1). Соседние диски связаны двумя гармоническими пружинами длиной $r_{0} \geq r_{s}$ и жесткостью $k$. Каждый диск характеризуется углом поворота $\varphi_{k}$ вокруг оси $Z$. Гамильтониан цепочки из $N$ дисков может быть представлен в виде

$$
\begin{gathered}
H=\sum_{k=1}^{N} \frac{I}{2} \dot{\varphi}_{k}^{2}+\sum_{k=1}^{N-1} U\left(\varphi_{k+1}-\varphi_{k}\right), \\
U(\Delta \varphi)=k\left(\sqrt{r_{s}^{2}+2 R^{2}(1-\cos (\Delta \varphi))}-r_{0}\right)^{2},
\end{gathered}
$$

где $I$ - момент инерции диска.

Потенциальная энергия взаимодействия двух соседних мономеров $U(\Delta \varphi)$ является $2 \pi$-периодической функцией и имеет два вырожденных положения равновесия при углах поворота $\Delta \varphi= \pm \varphi_{0}$, где $\varphi_{0}=\arccos \left(\left(2 R^{2}-r_{0}^{2}+r_{s}^{2}\right) / 2 R^{2}\right)$. Поскольку переход 
между этими положениями равновесия приводит к изменению конформации цепи, в дальнейшем мы будем говорить о них как о топологически неэквивалентных в отличие от положений равновесия, отличающихся на величину $\pm 2 \pi n$, которые являются топологически эквивалентными (см. рис. 2). Выберем значение потенциальной энергии при $\Delta \varphi=0$ (т. е., высоту барьера, разделяющего топологически неэквивалентные состояния) в качестве независимого параметра: $p=U(0)$. Полагая для удобства $U( \pm \pi)=1$ и $U\left( \pm \varphi_{0}\right)=0$, для параметров потенциала получим следующие соотношения:

$$
\begin{gathered}
r_{0}=r_{s}+\sqrt{p / k}, \\
R=\frac{1}{2 \sqrt{k}} \sqrt{(\sqrt{U(\pi)}+\sqrt{p})\left(\sqrt{U(\pi)}+\sqrt{p}+2 r_{s} \sqrt{k}\right)} .
\end{gathered}
$$

Остальные параметры $\left(r_{s}, k, I\right)$ положим равными единице. После подстановки этих соотношений гамильтониан принимает вид

$$
\begin{aligned}
H & =\sum_{k=1}^{N} \frac{1}{2} \dot{\varphi}_{k}^{2}+\sum_{k=1}^{N-1} U\left(\varphi_{k+1}-\varphi_{k}\right), \\
U(\Delta \varphi)= & \left(\sqrt{1+\frac{1}{2}(1+\sqrt{p})(2+\sqrt{p})(1-\cos (\Delta \varphi))}\right. \\
& -(1+\sqrt{p}))^{2} .
\end{aligned}
$$

\section{3. Методика расчетов}

Рассмотрим цепь длины $N_{0}, N_{T}$ крайних дисков которые (слева и справа, соответственно) помещены в термостаты Ланжевена с температурами $T_{ \pm}=(1 \pm 0.1) T$. Зададим начальное условие, в котором положения частиц соответствуют одному из положений равновесия $\varphi_{n}(t=0)=(n-1) \cdot \varphi_{0}, n \in \overline{1, N_{0}}$, а распределение скоростей соответствует равновесному распределению при температуре $T$. Для крайних частиц системы предполагаем свободные граничные условия. Таким образом, динамика системы определяется следующими уравнениями движения

$$
\left\{\begin{array}{l}
\ddot{\varphi}_{n}=-\frac{\partial H}{\partial \varphi_{n}}-\gamma \dot{\varphi}_{n}+\sqrt{2 \gamma k_{\mathrm{B}} T_{+}} \eta_{n}(T), n \in \overline{1, N_{T}} \\
\ddot{\varphi}_{n}=-\frac{\partial H}{\partial \varphi_{n}}, n \in \overline{N_{T}+1, N_{0}-N_{T}}, \\
\ddot{\varphi}_{n}=-\frac{\partial H}{\partial \varphi_{n}}-\gamma \dot{\varphi}_{n}+\sqrt{2 \gamma k_{\mathrm{B}} T_{-}} \eta_{n}(T), n \in \overline{N_{0}-N_{T}+1, N_{0}}
\end{array}\right.
$$

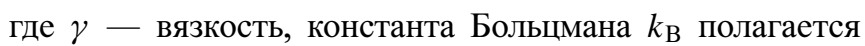
равной единице, а для случайного процесса $\eta_{n}(t)$ выполняются соотношения

$$
\left\langle\eta_{k}(t)\right\rangle=0, \quad\left\langle\eta_{k_{1}}\left(t_{1}\right) \eta_{k_{2}}\left(t_{2}\right)\right\rangle=\delta_{k_{1}, k_{2}} \delta\left(t_{1}-t_{2}\right) .
$$

Для интегрирования уравнений движения использовался алгоритм Верле с шагом интегрирования 0.05 в безразмерных единицах времени. Продолжительность расчета составляла $10^{8}-3 \cdot 10^{8}$ шагов. В ходе расчета в системе устанавливается стационарное состояние, в котором средние по времени величины локальной температуры $T_{n}$ и теплового потока $J_{n}$ достигают постоянных значений

$$
\begin{gathered}
T_{n}=\left\langle\dot{\varphi}_{n}^{2}\right\rangle, \\
J_{n}=-\left\langle\frac{1}{2}\left(\dot{\varphi}_{n}+\dot{\varphi}_{n-1}\right) \frac{\partial U}{\partial \varphi_{n}}\right\rangle .
\end{gathered}
$$

В области между термостатами устанавливается постоянный поток энергии $J$, не зависящий от номера частицы: $J=J_{n}, n \in \overline{N_{T}+1, N_{0}-N_{T}}$. Указанные выше величины используются для расчета коэффициента теплопроводности $\kappa$ :

$$
\kappa(N, T)=\frac{J(N-1)}{\Delta T},
$$

где $\Delta T=T_{N_{T}+1}-T_{N_{0}-N_{T}+1}-$ изменение температуры в средней области, $N=N_{0}-2 N_{T}-$ количество частиц между термостатами, $T$ - полусумма температур левого и правого термостатов.

Важной характеристикой, непосредственно влияющей на теплопроводность, является удельная теплоемкость $C$ :

$$
C(T)=\frac{1}{N} \frac{\partial\langle E\rangle}{\partial T},
$$

где $N-$ длина цепи, $\langle E\rangle-$ средняя полная энергия.

\section{4. Результаты и обсуждение}

Результаты моделирования теплопроводности цепочки длиной 60 ротаторов с различными величинами барьеров показаны на рис. 3-7.

На рис. 3 показана зависимость коэффициента теплопроводности от температуры системы для потенциалов с различными величинами „внутреннего“ барьера, разделяющего состояния $\pm \varphi_{0}$. Видно, что поведение коэффициента теплопроводности при низких температурах $(T<0.3)$ существенно зависит от высоты барьера $p$. А именно, для малых величин $p(<0.05)$ эта зависимость носит немонотонный характер с максимумом при $T \sim 0.15$, тогда как при больших значениях $p$ эта немонотонность практически не наблюдается. Более того, при отсутствии барьера $(p=0)$ теплопроводность такой системы стремится к нулю при $T \rightarrow 0$ в отличие от предельного случая гармонической цепи, где она неограниченно растет с уменьшением температуры.

Рассмотрим этот предельный случай подробнее (см. рис. 4). На первый взгляд потенциал (2) при $p=0$ аналогичен исследованному в работе [21] потенциалу ротаторов $U(x) \sim 1-\cos x$ : система характеризуется 


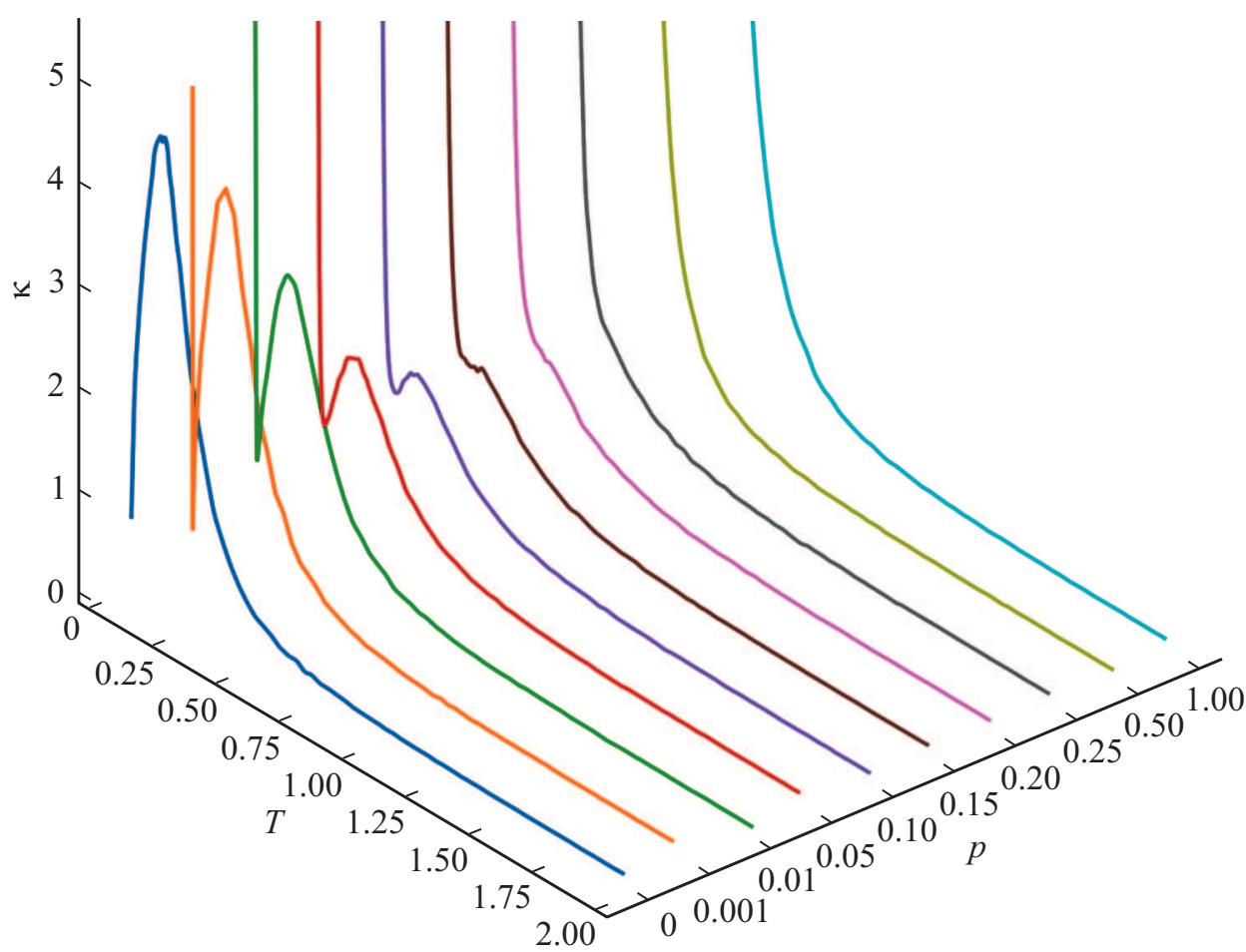

Рис. 3. Зависимость коэффициента теплопроводности $\kappa$ от температуры $T$ для различных величин высоты внутреннего барьера $p$.

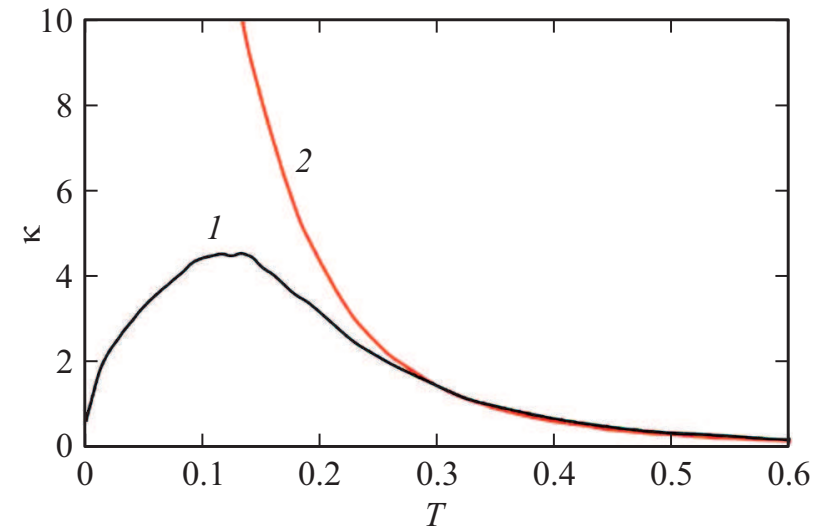

Рис. 4. Сравнение температурной зависимости коэффициента теплопроводности цепи с двумя различными потенциалами взаимодействия соседей. Кривая 1 соответствует модели двухбарьерных ротаторов при $p=0,2$ - модели ротаторов $U(x)=(1-\cos x) / 2$.

единственным положением равновесия и потенциальная энергия ограничена сверху. Однако, в этой работе показано, что в пределе низких температур $(T \rightarrow 0)$ коэффициент теплопроводности неограниченно растет, что соответствует предельному случаю системы связанных гармонических осцилляторов. В рассматриваемом нами случае теплопроводность стремится к нулю $\left(\lim _{T \rightarrow 0} \kappa=0\right)$, поскольку в системе с потенциалом (2) отсутствуют линейные элементарные возбуждения (фононы). Действительно, при $p=0$ разложе- ние потенциала (2) начинается с четвертого порядка $\left(U \sim\left(\varphi_{j+1}-\varphi_{j}\right)^{4}\right)$, поэтому в системе могут существовать только нелинейные волны (так называемый режим акустического вакуума). Специфика нелинейных элементарных возбуждений заключается в зависимости их частоты от амплитуды $A$ (в пределе малых амплитуд $\omega \sim A)$ [3]. Последнее обстоятельство приводит к тому, что групповая скорость, определяющая процесс переноса энергии, исчезает при $T \rightarrow 0: d \omega / d k \sim A \rightarrow 0$. Что же касается второй составляющей коэффициента теплопроводности - теплоемкости, то при нулевой температуре ее величина достигает некоторого значения несколько меньше единицы, как это показано на рис. 6 (кривая 1). Такое поведение также можно объяснить чисто нелинейным характером элементарных возбуждений. Действительно, полная энергия системы складывается из кинетической и потенциальной, средние значения которых в линейной системе равны. В рассматриваемом нами случае этот принцип нарушается и доминирует кинетическая энергия, поскольку ее величина пропорциональна квадрату смещения, а величина потенциальной энергии - только четвертой степени. Поэтому, несмотря на уменьшение частоты с понижением амплитуды колебаний, теплоемкость, как производная от полной энергии по температуре, оказывается меньше единицы $\left(C=\frac{d E}{d T} \leq 1\right)$.

C повышением температуры наблюдается быстрый рост теплопроводности, который связан как с увеличением частоты нелинейных волн, так и с ростом их групповой скорости: при температуре $T \sim 0.15$ коэффициент 

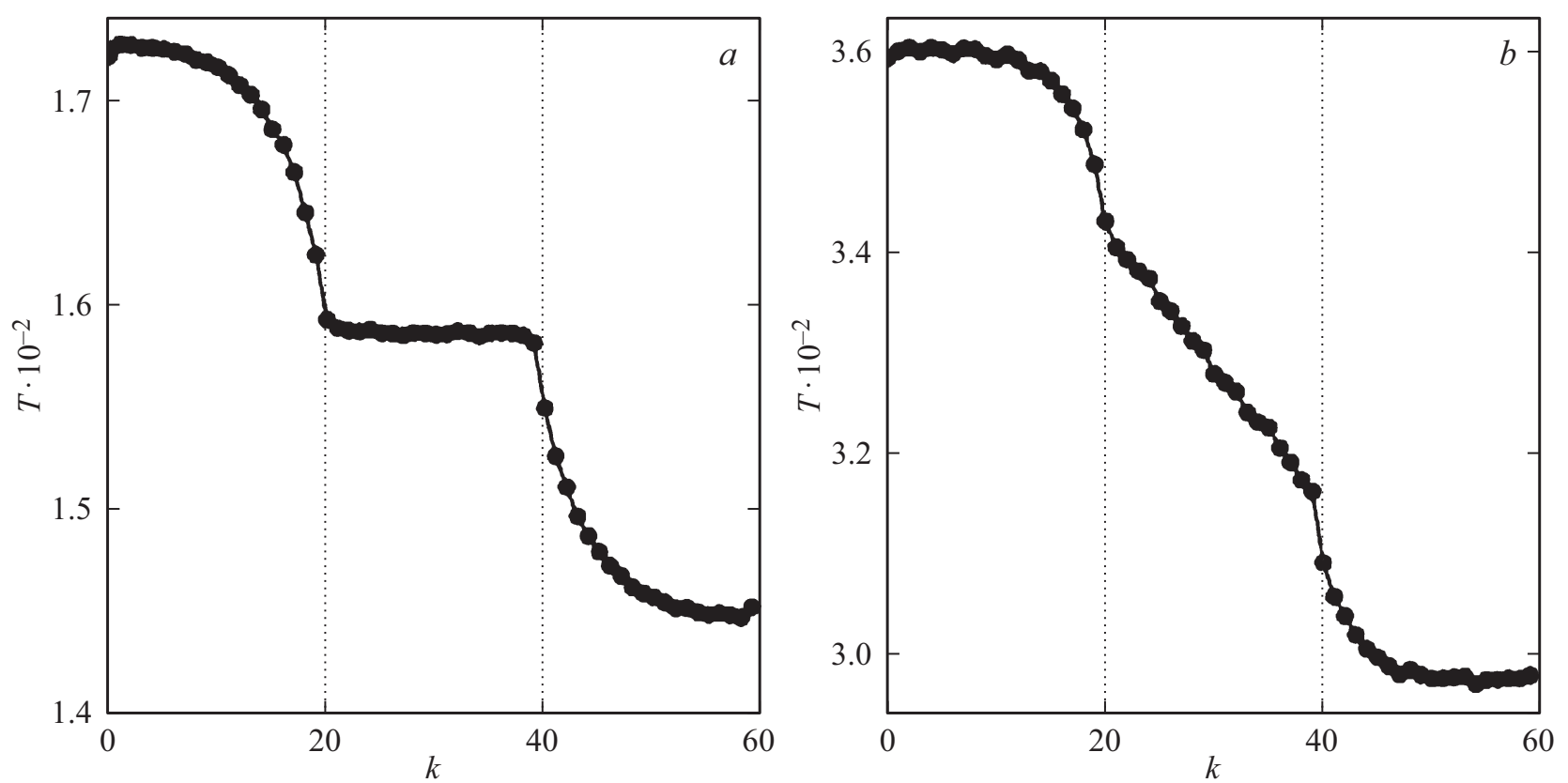

Рис. 5. Сравнение температурных профилей в $(a)$ модели ротаторов $U(x)=(1-\cos x) / 2$ и $(b)$ модели двухбарьерных ротаторов при $p=0$.

теплопроводности достигает максимального значения, после чего начинается достаточно быстрое падение и при температурах $T \sim 0.3$ его значение достигает величин, характерных для системы ротаторов [21]. Отсюда следует, что $T \sim 0.3$ обозначает условную границу, где вращательные флуктуации начинают вносить заметный вклад в процесс передачи энергии.

Второй важной особенностью нелинейных волн является их взаимодействие между собой, что, с одной стороны, вызывает эффективное перераспределение энергии между ними, а с другой стороны, может приводить к формированию локализованных нелинейных возбуждений. Последние могут служить переносчиками энергии, что приводит к баллистическому характеру теплопроводности (подобные процессы предсказаны в углеродных нанотрубках [22]), либо, наоборот, являются эффективными центрами рассеяния фононов (ротобризеры в цепочке ротаторов [21]). В связи с этим интересно рассмотреть температурный профиль рассматриваемой нами системы при низких температурах. На рис. 5 показано сравнение распределения температуры вдоль цепи в цепочке ротаторов [21] (левая панель) и в рассматриваемой нами системе при $p=0$ (правая панель). Видно, что для цепочки ротаторов это распределение аналогично распределению температуры в цепи гармонических осцилляторов, что обусловлено баллистическим переносом энергии линейными фононами в отсутствии каких-либо процессов рассеяния. Напротив, в рассматриваемой нами системе наблюдается практически линейное убывание температуры от более горячего термостата к более холодному. Это говорит о преимущественно диффузионном характере переноса энергии, вызванном взаимодействием между нелинейными волнами. Поскольку при низких температурах появление ротобризеров практически исключено, можно утверждать, что вклад от локализованных нелинейных возбуждений несущественен.

Возвращаясь к анализу поведения теплопроводности при малых (но не нулевых) величинах барьера $0<p<0.05$ на рис. 3, можно видеть, что нелинейность переносчиков энергии оказывается доминирующей в диапазоне температур $0<T<0.5$, исключая только малый интервал непосредственно вблизи $T=0$, где все-

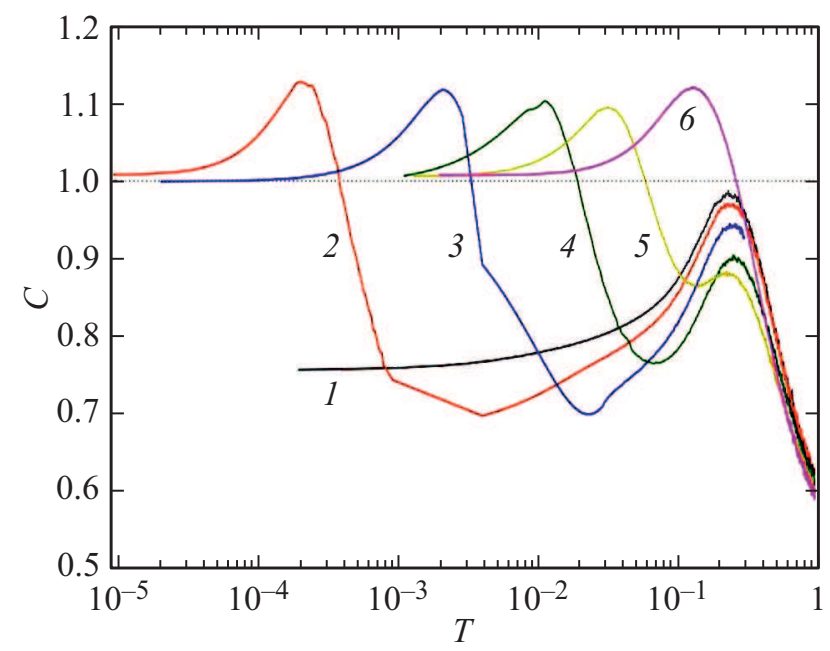

Рис. 6. Зависимость теплоемкости от температуры системы для различных величин барьера. Кривая 1 соответствует значению высоты внутреннего барьера $p=0,2-0.001,3-0.01$, $4-0.05,5-0.15,6-0.5$. 


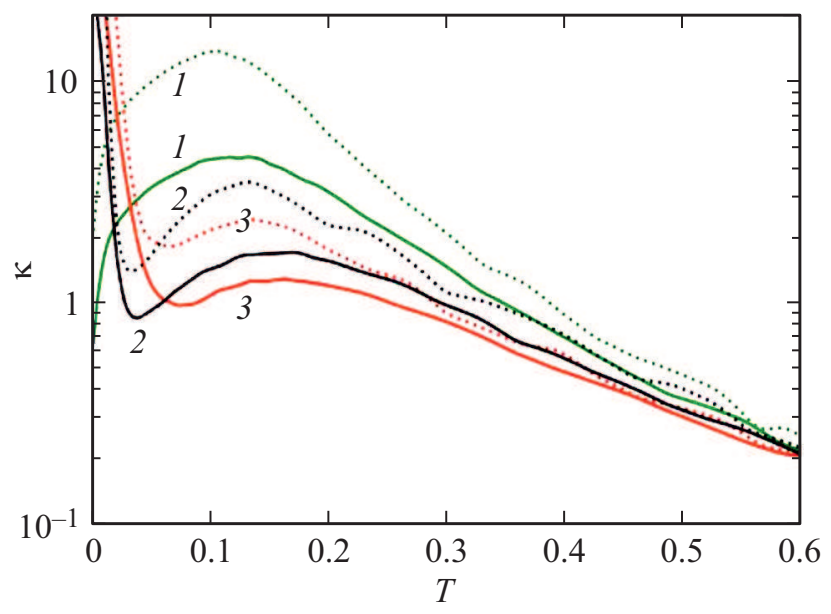

Рис. 7. Зависимость коэффициента теплопроводности от температуры для цепей с различными длинами и высотой внутреннего барьера. Кривые 1 соответствуют высоте внутреннего барьера $p=0,2-0.05,3-0.1$. Сплошные линии соответствуют коротким цепям $(N=60)$, пунктирные - длинным $(N=600)$.

таки присутствуют линейные фононы. О доминировании нелинейных процессов в данном диапазоне высот барьера, разделяющего топологически неэквивалентные состояния, говорит и поведение теплоемкости на рис. 6, где хорошо видно, что при малых значениях $p$ теплоемкость системы падает приблизительно до одного и того же значения $C \sim 0.75$, характерного для потенциала c $p=0$.

При больших значениях высоты барьера линейные или квазилинейные фононы доминируют в области малых температур, обеспечивая рост коэффициента теплопроводности при $T \rightarrow 0$ (рис. 3). При более высоких температурах существенную роль начинают играть процессы надбарьерных переходов, а затем и локальные (но не локализованные) вращательные флуктуации. В пределе высоких температур $(T>1)$ большая часть цепочки переходит во вращательное движение, блокируя тем самым перенос тепла.

В заключение следует отметить, что основные результаты остаются верны как в случае фиксированных граничных условий, так и при увеличении длины цепи до $N=600$ (см. рис. 7). При этом разница между минимальным и максимальным значением $\kappa$ несколько увеличивается с ростом длины цепи.

Таким образом, результаты моделирования показывают, что в рассмотренной системе при малых значениях внутреннего барьера, разделяющего топологически неэквивалентные состояния, определяющую роль играют нелинейные волны. Их взаимодействие обеспечивает практически диффузионный процесс переноса энергии, что приводит к линейному профилю распределения температур вдоль цепи. При ненулевых значениях высоты барьера линейные и квазилинейные фононы также не вносят заметного вклада в процесс теплопроводности, исключая малый интервал температур непосредственно вблизи $T=0$. В области промежуточных и высоких температур на процессы переноса тепла эффективно влияют надбарьерные переходы, что приводит к уменьшению теплопроводности цепи.

\section{5. Заключение}

В настоящей работе рассмотрена механическая модель для описания процессов переноса энергии в полимерных системах, в которых вращательное движение мономеров играет существенную роль. Примерами таких систем являются изолированные цепочки полиэтилена в вытянутом состоянии [23], отдельные молекулы парафинов в кристалле [24], а также супрамолекулярные структуры [25]. В отличие от большинства предыдущих работ [19-21], в модели учитывается топология конформационной подвижности, то есть наличие нескольких устойчивых состояний. Показано, что наличие двух минимумов в периодическом потенциале взаимодействия соседей приводит к немонотонной зависимости теплоемкости цепи и коэффициента теплопроводности от температуры в том случае, когда высоты барьеров, разделяющих топологически эквивалентные и неэквивалентные состояния, различаются более чем в пять раз. При больших значениях высоты внутреннего барьера наблюдается монотонное уменьшение коэффициента теплопроводности, характерное для одномерных систем с ограниченными потенциалами взаимодействия. Сравнение результатов со случаем, когда внутренний барьер отсутствует (при котором реализуется режим акустического вакуума), указывает на преобладающий характер нелинейных волн по сравнению с линейными фононами. Вследствие этого при небольшой высоте внутреннего барьера наблюдается рост коэффициента теплопроводности.

Теоретически, такое поведение может служить основой для управления теплопроводностью цепи, если с помощью внешнего воздействия удается изменять относительные высоты барьеров.

\section{Благодарности}

Авторы благодарят Ю.А. Косевича за участие в плодотворных обсуждениях.

\section{Финансирование работы}

Научно-исследовательская работа выполнена за счет субсидии, выделенной ФИЦ ХФ РАН на выполнение государственного задания, тема 0082-2014-0013 (номер АААА544 А17-117042510268-5) и при финансовой поддержке РФФИ в рамках научного проекта 20-3390165. Вычислительные ресурсы предоставлены межведомственным суперкомпьютерным центром РАН. 


\section{Конфликт интересов}

Авторы заявляют, что у них нет конфликта интересов.

\section{Список литературы}

[1] T.M. Tritt. Thermal Conductivity: Theory, properties and apllications. Springer US (2004). 290 c.

[2] E. Fermi, J. Pasta, S. Ulam. LASL Rep. LA-1940 (1955).

[3] A. Scott. Nonlinear Science Emergence and Dynamics of Coherent Structures. Oxford University Press (2003). 504 c.

[4] S. Iijima. Nature 354, 6348, 56 (1991).

[5] A.K. Geim, K.S. Novoselov. Nature Mater. 6, 3, 183 (2007).

[6] O.M. Braun, Y.S. Kivshar. The Frenkel-Kontorova Model. Springer-Verlag (2004). $472 \mathrm{c}$.

[7] G.M. Sagdeev, R.Z. Usikov, D.A. Zaslavsky. Nonlinear Physics - From the Pendulum to Turbulence and Chaos. Harwood Academic (1990). 675 c.

[8] S. Lepri, R. Livi, A. Politi. Phys. Rep. 377, 1, 1 (2003).

[9] A. Dhar. Adv. Phys. 57, 5, 457 (2008).

[10] S. Lepri, R. Livi, A. Politi. PRL 125, 4, 040604 (2020).

[11] C. Giardiná, R. Livi, A. Politi, M. Vassalli. PRL 84, 10, 2144 (2000).

[12] O.V. Gendelman, A.V. Savin. PRL 84, 11, 2381 (2000).

[13] A.V. Savin, Y.A. Kosevich. Phys. Rev. E 89, 3, 032102 (2014).

[14] D. Xiong. J. Status Mech. Theory Exp. 2016, 4, 043208 (2016).

[15] D. Roy. Phys. Rev. E 86, 4, 041102 (2012).

[16] G.R. Archana, D. Barik. Phys. Rev. E 99, 2, 022103 (2019).

[17] A.V. Savin, O.V. Gendelman. Phys. Rev. E 89, 1, 012134 (2014).

[18] V.B. Pinheiro, P. Holliger. Curr. Opin. Chem. Biol. 16, 3-4, 245 (2012).

[19] S. Homma, S. Takeno. Prog. Theor. Phys. 72, 4, 679 (1984).

[20] S. Takeno, M. Peyrard. Phys. D 92, 3-4, 140 (1996).

[21] А.В. Савин, О.В. Гендельман. ФТТ 43, 2, 341 (2001).

[22] C. Yu, L. Shi, Z. Yao, D. Li, A. Majumdar. Nano Lett. 5, 9 , 1842 (2005).

[23] N.I. Rubtsova, C.M. Nyby, H. Zhang, B. Zhang, X. Zhou, J. Jayawickramarajah, A.L. Burin, I.V. Rubtsov. J. Chem. Phys. 142, 21, 212412 (2015).

[24] M.A. Kovaleva, V.V. Smirnov, L.I. Manevitch. Mater. Phys. Mech. 35, 1, 80 (2018).

[25] A.V. Gorbunov, T. Putzeys, I. Urbanavičiūtè, R.A.J. Janssen, M. Wübbenhorst, R.P. Sijbesma, M. Kemerink. Phys. Chem. Chem. Phys. 18, 34, 23663 (2016).

Редактор Т.Н. Василевская 